\title{
DETERMINACIÓN DE LAS PROPIEDADES MECÁNICAS DE LA CAÑA GUADUA ANGUSTIFOLIA DEL ECUADOR CUANDO ESTÁ SOMETIDA A FUERZAS AXIALES
}

\author{
DETERMINATION OF THE MECHANICAL PROPERTIES OF GUADUA \\ ANGUSTIFOLIA CANE WHEN SUBJECTED TO AXIAL FORCES
}

\section{ANTONIO LA TEGOLA', LUIS OCTAVIO YÉPEZ ROCA², WALTER VICENTE MERA ORTIZ², PEDRO LUIS CÓRDOVA ALCÍVAR ${ }^{4}$}

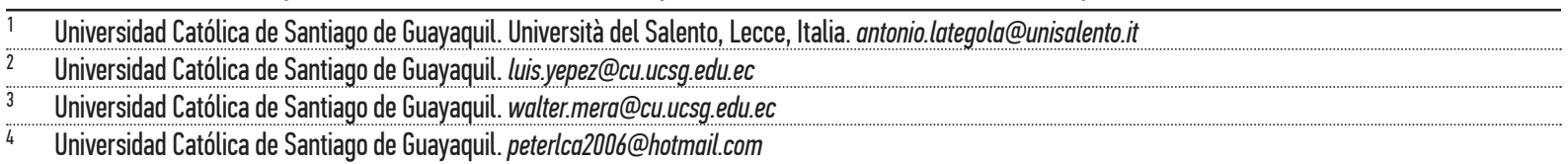

RESUMEN

El objetivo de este trabajo de investigación es la determinación de las propiedades mecánicas de la caña Guadua Angustifolia Kunth de Ecuador, y a través de la metodología experimental determinar los valores de la resistencia a la compresión, a la tracción y su módulo de elasticidad. Se realizaron ensayos estructurales a muestras de caña Guadua obtenidas de plantaciones de Valencia, provincia de Los Ríos, Ecuador para dar un soporte ingenieril y científico a este trabajo. Las muestras fueron sometidas a ensayos de compresión y tracción. La metodología cuantitativa dio base al trabajo empírico de la investigación, para el cual se determinaron cuatro variables independientes que fueron ajustadas a través de la revisión bibliográfica. La metodología de diseño se basó en la mecánica estructural y la norma ISO 22157. El conjunto de las variables será explicado a través del estudio de la información estadística. Los resultados de los ensayos permitieron determinar los valores característicos de las variables de estudio, con los cuales se pueden diseñar y construir estructuras de caña Guadua seguras. Con estos resultados se espera aportar a la creación de la nueva norma ecuatoriana de construcción y diseño estructural de bambú.

PALABRAS CLAVE: propiedades mecánicas, caña guadua Angustifolia Kunth, propiedades geométricas.
ABSTRACT

The objective of this research is to determine the mechanical properties of the Bamboo Guadua Angustifolia Kunth of Ecuador, and through the experimental methodology determine the values of the resistance to compression, tension and its modulus of elasticity. Structural tests were made to guadua samples obtained from plantations in Valencia, province of Los Ríos, Ecuador, to provide an engineering and scientific support to these work trials. The samples were subjected to compressive stress and tension. The quantitative methodology given to empirical research work consists of four independent variables and adjusted through the literature review. The design methodology is based on structural mechanics, and the International Standard ISO 22157. The set of variables will be explained through the study of statistical information. The test results allowed determining the characteristic values of the study variables, with which you can design and build safe structures of Guadua cane. According these results produced, will contribute to the creation of the new Ecuadorian standard construction and structural design of bamboo.

KEYWORDS: mechanical properties, bamboo Guadua Angustifolia Kunth, geometric properties. 
INTRODUCCIÓN

Conscientes del uso estructural de la caña guadua, en los últimos años se han desarrollado estudios en diferentes regiones y lugares donde ésta es cultivada para determinar sus propiedades y comportamiento mecánico para la correspondiente aplicación en sistemas estructurales.

Bajo la premisa de tecnificación del análisis y diseño de construcciones en guadua, se ha considerado pertinente llevar a cabo esta investigación para posteriormente comentar resultados y sugerir recomendaciones de análisis y diseño a ingenieros civiles y arquitectos.

El problema que se plantea resolver es el uso del bambú a través del conocimiento a través de pruebas de laboratorio de sus propiedades mecánicas y estructurales para la elaboración de un procedimiento que permita el uso de este material en el diseño, en el cálculo estructural y en la construcción de viviendas.

Es necesario investigar las propiedades mecánicas y estructurales del bambú para la elaboración de una norma de construcción que actualmente no existe en el país y que permita el uso seguro del bambú en la construcción de edificaciones.

La norma de construcción para el Ecuador propuesta para el bambú tiene relación con otras, tales como las normas del hormigón armado, acero y madera en general, y su elaboración y creación será de beneficio para los usuarios de la Norma Ecuatoriana de Construcción (NEC).

Las variables a medir son: propiedades del bambú (caña guadua) a los esfuerzos de compresión (esfuerzo axial) y tracción (esfuerzo axial); módulo elástico del bambú; clasificación de la variación de dimensiones de los especímenes del bambú: ancho, espesor, nudos, etc.

Los valores característicos de la resistencia se calcularon como percentiles de resistencia menores al 5\%. Teniendo en cuenta el número de muestras, se hizo referencia a la teoría de pequeñas muestras mediante la estimación de la media y de la varianza. Además. se adoptó un intervalo de confianza igual al $75 \%$ con el fin de tener en cuenta la naturaleza del material.

\section{METODOLOGÍA}

En los ensayos fueron usadas cañas guadua Angustifolia Kunth de Ecuador, traídas desde plantaciones cercanas al cantón Valencia, en la provincia de Los Ríos. Otros detalles sobre el origen de estas cañas son desconocidos pues ya estaban cortadas en longitudes de aproxima- damente seis metros. Habían sido tratadas en soluciones químicas con bórax y ácido bórico, y sometidas a procedimientos de secado para mejorar su durabilidad. Estas cañas fueron almacenadas en bodegas en los galpones de la planta de Ecomateriales de la Universidad Católica de Santiago de Guayaquil (UCSG) en Durán.

Alrededor de 170 cañas guadua aparentemente con tallos sanos, sin ningún tipo de ataque por insectos o de hongos, huecos o imperfecciones, se seleccionaron arbitrariamente más de diez cañas para hacer las muestras de los ensayos.

Referentes a la tracción, se elaboraron doce muestras y para las pruebas de compresión se elaboraron catorce muestras. Las pruebas de los ensayos se realizaron en el laboratorio de estructuras de la Facultad de Ingeniería de la UCSG. en el edificio del Centro de Investigaciones en Ingeniería Estructural Sismo resistente (CEINVES). Se tuvieron que realizar algunas adaptaciones a los equipos existentes debido a que era la primera vez que se hacía este tipo de pruebas.

\section{PREPARACIÓN DE EQUIPOS DE ENSAYOS DE COMPRESIÓN}

Se elaboraron catorce muestras de caña guadua para estos ensayos, la altura de cada muestra era igual a su medida de diámetro externo, es decir, una relación 1:1. Las muestras no contenían nudos.

El ensayo consistió en aplicar fuerzas de compresión paralela a la fibra de la caña guadua en los extremos de las muestras a través de dos placas sólidas, tal como se puede apreciar en la Figura 1.
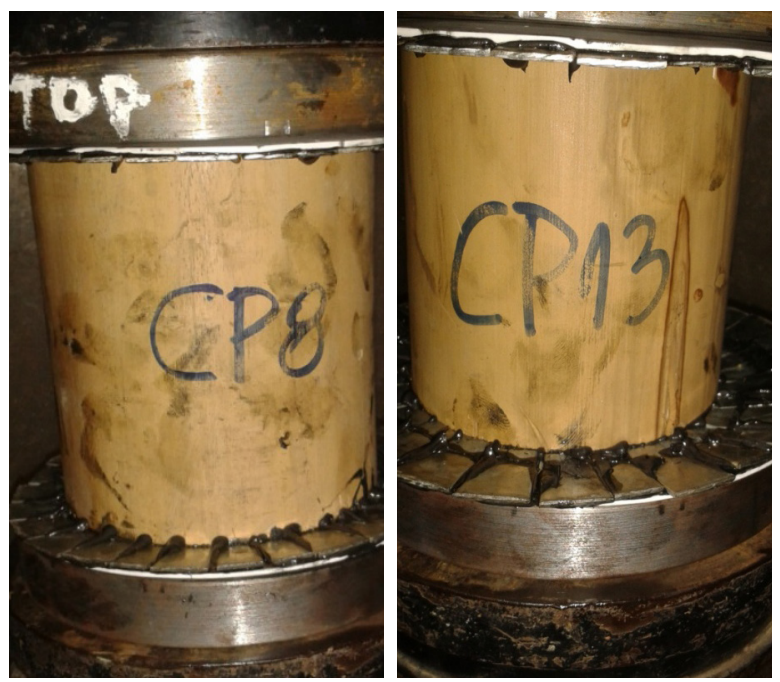

Figura 1. Ensayo de compresión simple paralela a la fibra. Fuente: Córdova. P. 2014. Obtención de las Propiedades mecánicas y estructurales de la caña guadua Angustifolia Kunth del Ecuador. 
En la máquina de compresión se aplicaron fuerzas de compresión a las placas (de hierro de 1 pulgada), sobre las cuales se asienta un disco de teflón de 0.0625 (1/16) pulgada de espesor, al que se aplica una capa fina de grasa grafitada en la que se asientan cuñas de lata de espesor pequeño según puede apreciarse en la Figura 2.
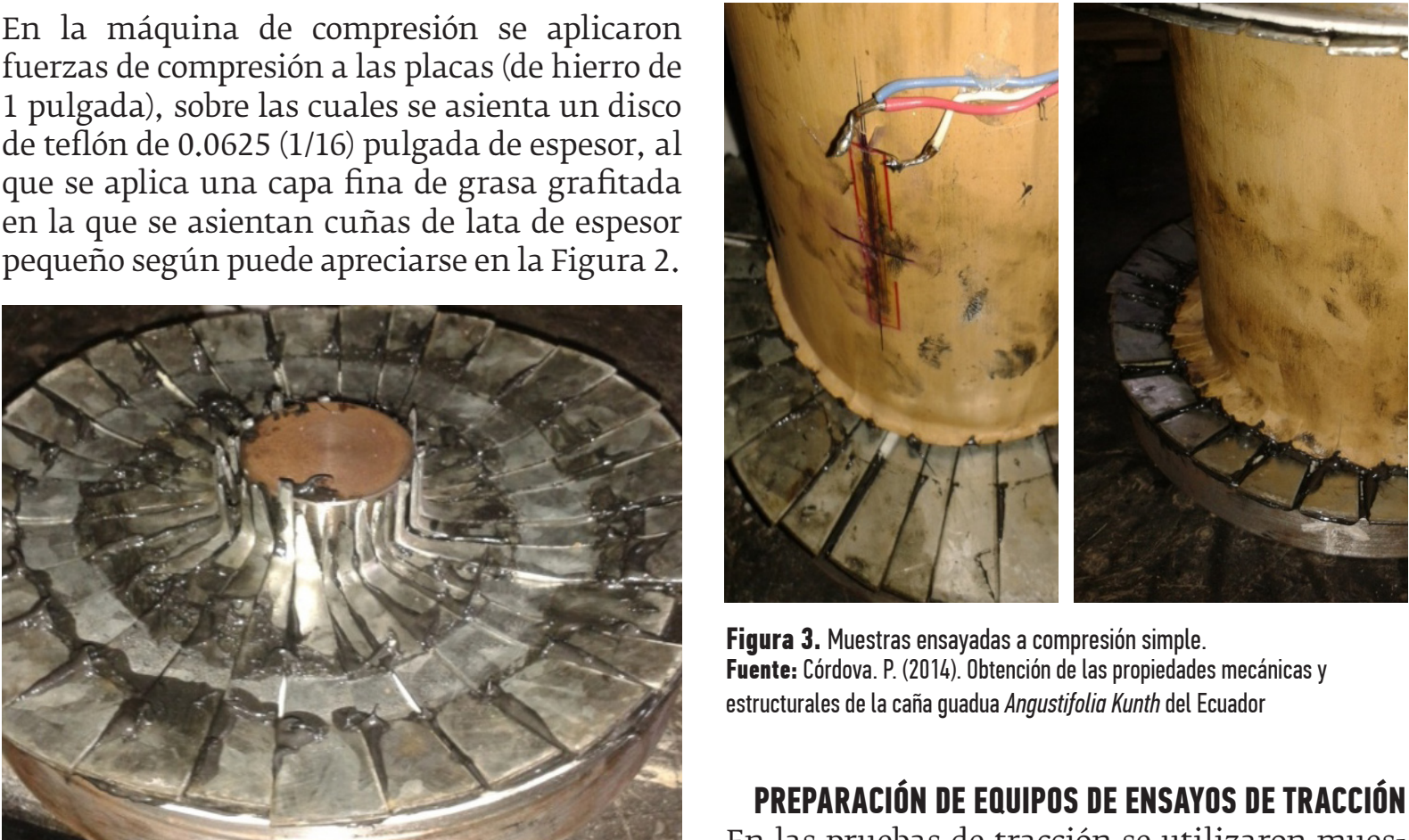

Figura 3. Muestras ensayadas a compresión simple. Fuente: Córdova. P. (2014). Obtención de las propiedades mecánicas y estructurales de la caña guadua Angustifolia Kunth del Ecuador

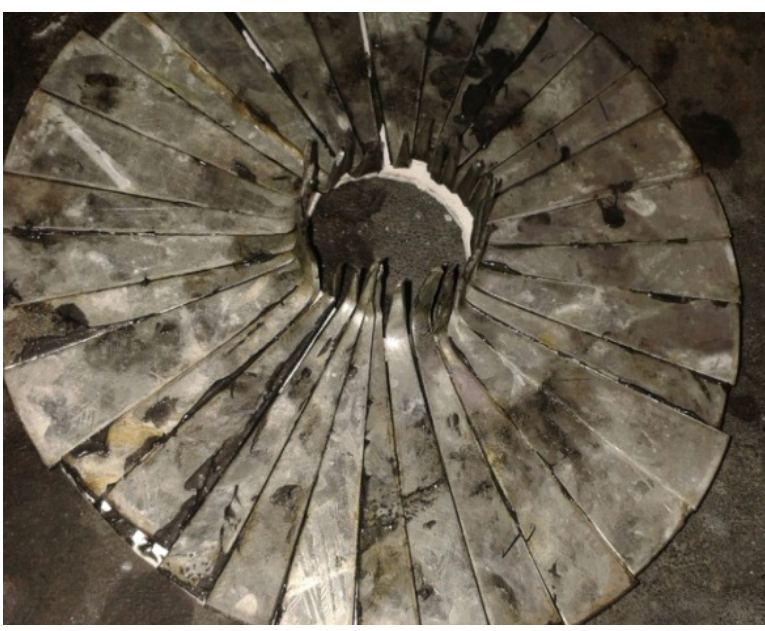

Figura 2. Placa elaborada para el ensayo de compresión libre de fricción en la muestra. que permite el deslizamiento horizontal.

Fuente: Córdova. P. 2014. Obtención de las Propiedades mecánicas y estructurales de la caña guadua Angustifolia Kunth del Ecuador.

Todo esto se realiza para que en los extremos de la muestra no haya fricción y no ocurra el fenómeno de confinamiento, es decir pueda existir desplazamiento horizontal.

El mecanismo de falla del ensayo de compresión al llegar a la resistencia última de la compresión paralela de la fibra se muestra en la Figura 3. Los strain gauges fueron instalados en las muestras de compresión para poder medir la deformación a medida que se aumentaba la carga y poder determinar el módulo de elasticidad de la caña guadua.

\section{PREPARACIÓN DE EQUIPOS DE ENSAYOS DE TRACCIÓN}

En las pruebas de tracción se utilizaron muestras de caña guadua ahusadas de $70 \mathrm{~cm}$ de largo. Se ensayaron 12 muestras con dichas características y además se midieron las propiedades de la sección de cada guadua de donde se extrajo la probeta.

Las muestras de tiras de caña guadua. fueron sometidas a una reducción de su área en el centro para el ensayo de tracción. La sección de medición tenía un área compuesta por el espesor de la pared de la caña y un ancho mayor a $1 \mathrm{~cm}$., además la longitud de la sección de medición era de $10 \mathrm{~cm}$. Todas las muestras tuvieron un nudo en la sección de medición. En la sección de medición se colocaron strain gauges para obtener las deformaciones que sufría la muestra a medida que se la sometía a la carga.

En los extremos se elaboraron los agarres para poder halar la muestra, que consistían en apretar los extremos de las muestras con placas metálicas empernadas y crear fricción entre la caña y las placas adhiriéndoles lijas.

Se esperaba que la resistencia a la tracción de la caña fuera menor que la fricción que ocurría en los extremos entre la lija pegada a la caña y la pegada a las placas, pero no fue así. Entonces se diseñó otra forma de agarre, que consistió en tuberías metálicas de $20 \mathrm{~cm}$. de largo, a las cuales se les soldó una abrazadera en cada extremo. Los extremos de las muestras fueron tallados para eliminar la superficie lisa y crear fricción, luego fueron insertados en el tubo y se vertió en el interior un epóxico para anclajes de Sika ${ }^{\circledR}$. 


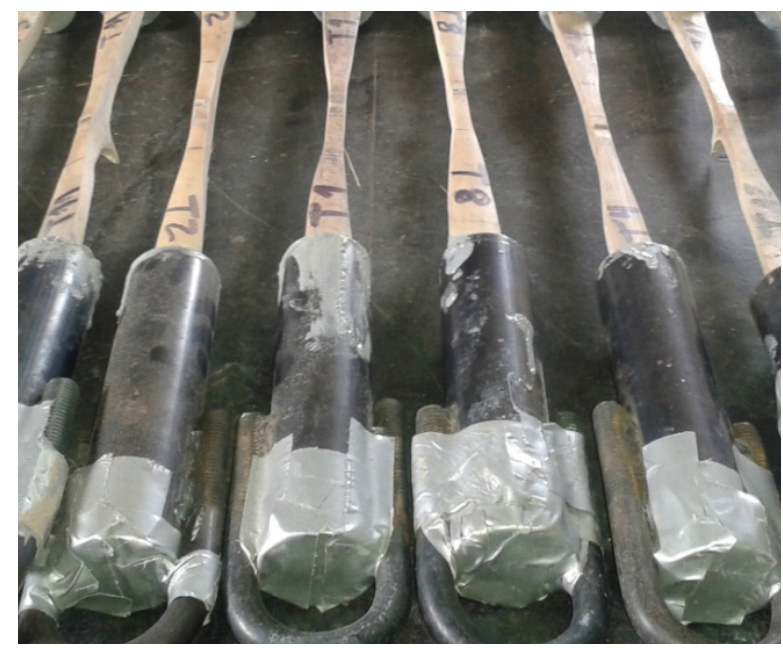

Figura 4. Muestras de tiras de caña guadua con una reducción de su área en el centro para el ensayo de tracción.

Fuente: Córdova. P. (2014). Obtención de las Propiedades mecánicas y estructurales de la caña guadua Angustifolia Kunth del Ecuador.
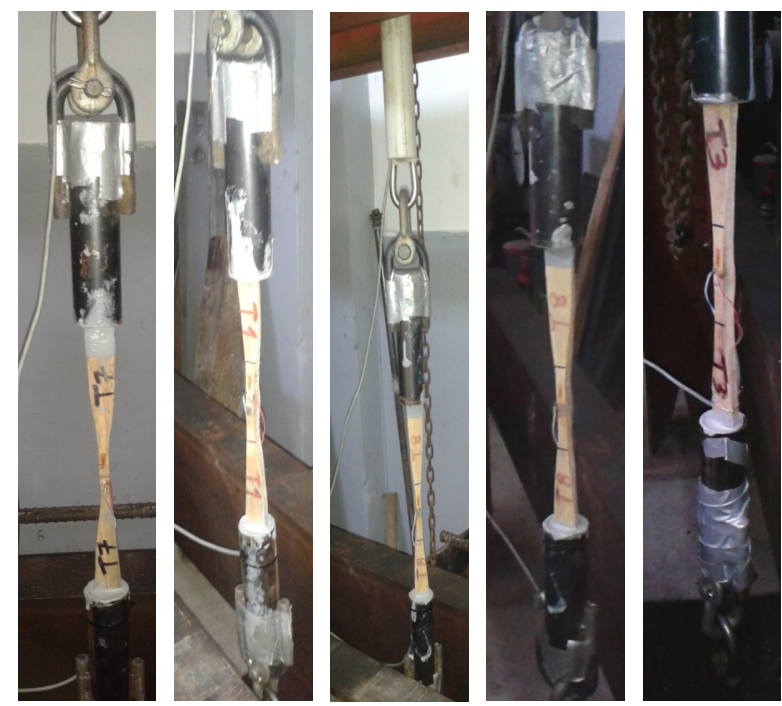

Figura 6. Ensayo de tracción paralela a la fibra.

Fuente: Córdova. P. (2014). Obtención de las Propiedades mecánicas y estructurales de la caña guadua Angustifolia Kunth del Ecuador.

Estos agarres dieron mejores resultados en las pruebas, siendo más resistentes a la tracción que la resistencia que otorgaba la muestra de caña en su sección de medición, tal como puede apreciarse en la Figura 4.

A través de una celda de carga se obtienen las lecturas de la fuerza que se va aplicando a la muestra y con los strain gauges se obtienen las lecturas de las deformaciones correspondientes a esas cargas, según puede apreciarse en la Figura 5.

Ambos extremos de las muestras se agarran con abrazaderas para poder halar aplicando

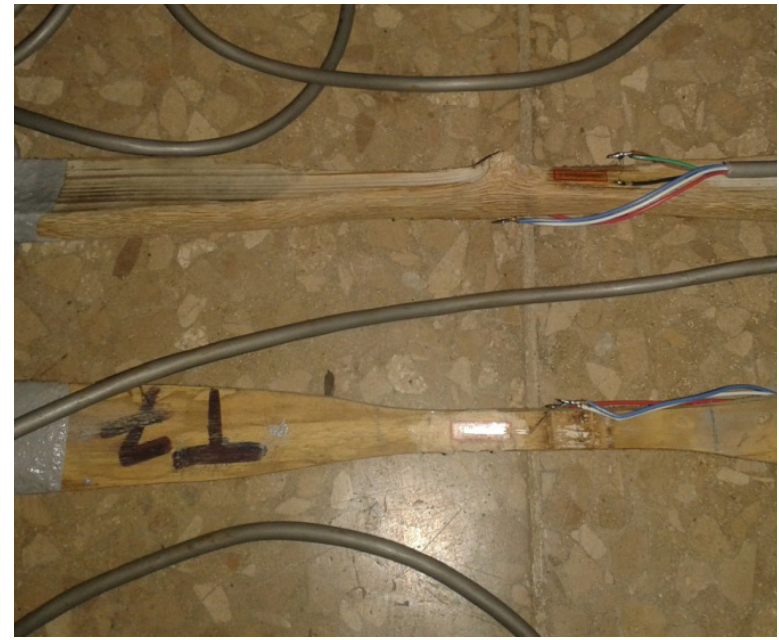

Figura 5. Strain gauges colocados en la sección de medición. Fuente: Córdova. P. (2014). Obtención de las propiedades mecánicas y estructurales de la caña guadua Angustifolia Kunth del Ecuador.

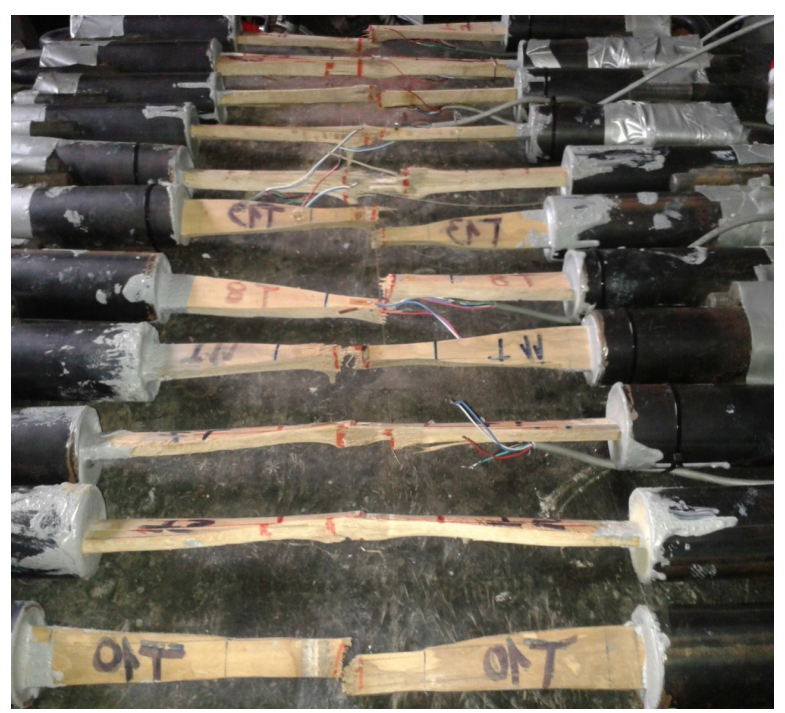

Figura 7. Mecanismo de falla de muestras de caña guadua a tracción. Fuente: Córdova. P. (2014). Obtención de las Propiedades mecánicas y estructurales de la caña guadua Angustifolia Kunth del Ecuador.

la carga de tracción a través de una polea de 3 toneladas de capacidad. Se aplican cargas de forma sucesiva hasta llegar a la falla de rotura por tracción, como se muestra en la Figura 6.

Todas las muestras fallaron en la sección de medición, como se tenía previsto, por tener las menores dimensiones. Además se observó que era una falla frágil, es decir instantánea. Se lograba escuchar cómo iban rompiéndose las primeras fibras para después desgarrarse bruscamente (Figura 7). 
RESULTADOS

PRUEBAS DE COMPRESIÓN PARALELA A LA FIBRA

A partir de los ensayos se ha determinado una resistencia última característica a la compresión de caña guadua valorada en $39.58 \mathrm{MPa}$, lo que da igual a $403.61 \mathrm{~kg} / \mathrm{cm}^{2}$.

Este valor es muy importante, puesto que si se lo compara con la resistencia del hormigón común de $280 \mathrm{~kg} / \mathrm{cm}^{2}$, podemos concluir que la caña guadua es un material de construcción, 1.44 veces más resistente a la compresión que el hormigón común. Esto es muy positivo, puesto que se obtiene mucha resistencia a muy bajo peso propio del material, lo que nos permite bajar la carga muerta en la estructura. Los resultados se muestran en las Tablas 1 y 2. Por su parte, la Figura 8 ilustra la varianza y distribución de frecuencia de los resultados de los ensayos.

Se obtuvieron las curvas esfuerzo-deformación unitaria de doce muestras sometidas a compresión paralela a la fibra. La Figura 9 ilustra la curva de la muestra 1 . De estas curvas podemos concluir que la caña guadua sometida a compresión paralela a la fibra se comporta como un material elástico. Calculamos su módulo de elasticidad al 50\% de la carga última, resultando en $16554 \mathrm{MPa}$, el cual es aceptable comparándolo con el Módulo de Elasticidad (MOE) del hormigón común, $24787 \mathrm{MPa}$. Estos resultados son mostrados en las Tablas 3 y 4.

Se obtuvo resultados relativamente uniformes para ser un material natural y primitivo, cultivado y cosechado en haciendas sin ningún tipo de desarrollo tecnológico para mejorar la resistencia de la caña o darle uniformidad a los resultados finales obtenidos. Se deben seguir desarrollando estudios para un mayor número de muestras. Un aspecto geométrico a destacar es que a medida que aumenta el espesor se aumenta la resistencia a la compresión.

TABLA 1. MUESTRAS DE LOS RESULTADOS DE LOS ENSAYOS Y CÁLCULO DEL ESFUERZO DE COMPRESIÓN ÚLTIMO DE CAÑA GUADUA

\begin{tabular}{rrrrrrrrrr} 
MUESTRA N & D EXT (CM) & D INT (CM) & ESP (CM) & PESO (G) & ÁREA (CM2) & P (KN) & OULT (MPA) & \multicolumn{1}{c}{ ESP/DEXT } & DM (CM) \\
\hline 1 & 9.84 & 6.49 & 1.59 & 269.8 & 42.966 & 169.5 & 39.450 & 0.1616 & 8.165 \\
\hline 2 & 10.23 & 8.55 & 0.88 & 189.1 & 24.78 & 127.6 & 51.494 & 0.0860 & 9.39 \\
\hline 3 & 9.65 & 8.05 & 0.8 & 176.1 & 22.242 & 127.3 & 57.233 & 0.0829 & 8.85 \\
\hline 4 & 10.84 & 9.23 & 0.76 & 229.2 & 25.378 & 127.1 & 50.082 & 0.0701 & 10.035 \\
\hline 5 & 9.83 & 8.19 & 0.77 & 202 & 23.211 & 123.7 & 53.294 & 0.0783 & 9.01 \\
\hline 6 & 10.69 & 9.96 & 0.85 & 221.2 & 25.284 & 132.8 & 52.523 & 0.0795 & 9.875 \\
\hline 7 & 9.81 & 8.12 & 0.82 & 173.7 & 23.799 & 112.1 & 47.103 & 0.0836 & 8.965 \\
\hline 8 & 11.19 & 9.05 & 1 & 265.6 & 34.018 & 168.7 & 49.591 & 0.0894 & 10.12 \\
\hline 9 & 10.44 & 8.81 & 0.85 & 187.1 & 24.644 & 106 & 43.013 & 0.0814 & 9.625 \\
10 & 10.06 & 8.25 & 0.83 & 182.7 & 26.029 & 122.7 & 47.140 & 0.0825 & 9.155 \\
\hline 11 & 10.14 & 7.11 & 1.45 & 268.4 & 41.051 & 185.5 & 45.188 & 0.1430 & 8.625 \\
12 & 9.8 & 8.16 & 0.9 & 188 & 23.133 & 114.5 & 49.495 & 0.0918 & 8.98 \\
\hline 13 & 9.64 & 6.82 & 1.4 & 243.9 & 36.456 & 171.5 & 47.043 & 0.1452 & 8.23 \\
\hline 14 & 10.32 & 6.87 & 1.56 & 290.9 & 46.578 & 182.8 & 39.246 & 0.1512 & 8.595 \\
\hline
\end{tabular}

Fuente: Córdova, P. (2014). Obtención de las propiedades mecánicas y estructurales de la caña guadua Angustifolia Kunth del Ecuador.

TABLA 2. ANÁLISIS ESTADÍSTICO DEL ESFUERZO DE COMPRESIÓN ÚLTIMO DE CAÑA GUADUA.

\begin{tabular}{lrl}
\hline o ULT MEDIA $=$ & 47.993 & Mpa \\
\hline Desviación estantadar $(S)=$ & 5.114 & Mpa \\
ok $=$ & 36.764 & Mpa \\
S/o ult Media= & 0.107 & \\
\hline
\end{tabular}

Fuente: Córdova, P. (2014). Obtención de las propiedades mecánicas y estructurales de la caña guadua Angustifolia Kunth del Ecuador.

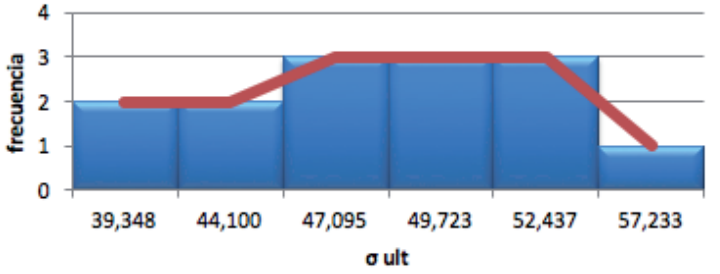

Figura 8: Análisis estadístico del esfuerzo último a compresión paralela a la fibra de la caña guadua. Fuente: Córdova, P. (2014). Obtención de las propiedades mecánicas y estructurales de la caña guadua Angustifolia Kunth del Ecuador. 
TABLA 3. CÁLCULO DEL MÓDULO DE ELASTICIDAD DE CAÑA GUADUA SOMETIDA A COMPRESIÓN PARALELA A LA FIBRA

\begin{tabular}{|c|c|c|c|}
\hline MUESTRA N & $\begin{array}{l}0.5[\text { ULT } \\
\text { (MPA) }\end{array}$ & $\begin{array}{l}\text { ME } \\
\text { (0.50ULT) }\end{array}$ & $\begin{array}{l}=(0.5[\text { ULT }) /(E(0.5 \Sigma U L T)) \\
(G P)\end{array}$ \\
\hline 1 & 71.631 & 5043 & 14.206 \\
\hline 2 & 69.195 & 4774 & 14.495 \\
\hline 3 & 70.465 & 4236 & 16,635 \\
\hline 4 & 68.200 & 5440 & 12.537 \\
\hline 6 & 69.348 & 3313 & 20.930 \\
\hline 7 & 61.806 & 2297 & 26.910 \\
\hline 8 & 80.637 & 3373 & 23.910 \\
\hline 10 & 64.257 & 3484 & 18.452 \\
\hline 11 & 65.309 & 4586 & 14.242 \\
\hline 12 & 74.078 & 3400 & 21.788 \\
\hline
\end{tabular}

Fuente: Córdova, P. (2014). Obtención de las propiedades mecánicas y estructurales de la caña guadua Angustifolia Kunth del Ecuador.

\begin{tabular}{|c|c|}
\hline o ULT MEDIA= & 25.525 \\
\hline Desviación estantadar $(\mathrm{S})=$ & 5.453 \\
\hline$E k=$ & 14.346 \\
\hline S/E ult Media= & 0.214 \\
\hline
\end{tabular}

Fuente: Córdova, P. (2014). Obtención de las propiedades mecánicas y estructurales de la caña guadua Angustifolia Kunth del Ecuador.

\section{PRUEBAS DE TRACCIÓN PARALELA A LA FIBRA}

Mediante ensayos se ha determinado la resistencia última característica a la tracción de la caña guadua valorada en $117.6 \mathrm{MPa}$; es decir, $1198.8 \mathrm{~kg} / \mathrm{cm}^{2}$, el cual comparándolo con el esfuerzo de fluencia del acero $4200 \mathrm{~kg} / \mathrm{cm}^{2}$, resulta aproximadamente 3.5 veces menos fuerte que el acero a tracción. Esto es muy positivo porque el acero es hecho de manera industrial por normas de calidad, además pasa por algunos procesos y necesita de mucha materia prima y mano de obra para poder ser elaborado. Se debe considerar que en la caña guadua se obtiene alta resistencia a la tracción con un bajo peso propio y además a un bajo costo. Los resultados de los ensayos se muestran en la Tabla 5 y el análisis estadístico puede verse en la Tabla 6. De igual forma en la Figura 10 se representa la varianza producto de los resultados.
(CP1) $\sigma(\mathrm{Mpa}) / \delta$

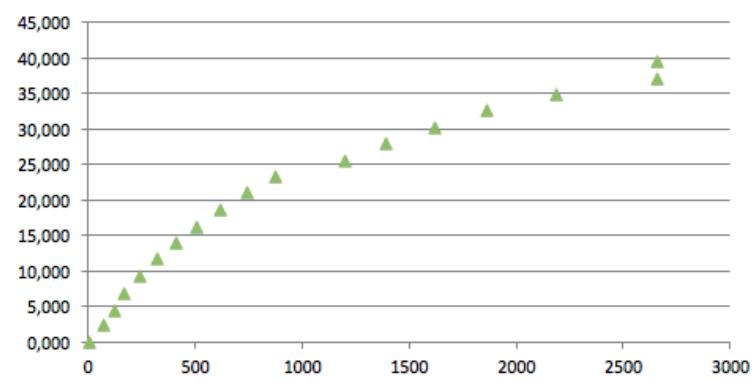

Figura 9. Curva del esfuerzo de compresión paralela a la fibra vs. la deformación unitaria de la muestra CP1.

Fuente: Córdova, P. (2014). Obtención de las propiedades mecánicas y estructurales de la caña guadua Angustifolia Kunth del Ecuador.

TABLA 5. MUESTRAS DE LOS RESULTADOS DE LOS ENSAYOS Y CÁLCULO DEL ESFUERZO DE TRACCIÓN ÚLTIMA DE CAÑA GUADUA

\begin{tabular}{|c|c|c|c|c|c|}
\hline MUESTRA & $\begin{array}{r}\text { ESPESOR } \\
\text { (CM) }\end{array}$ & $\begin{array}{r}\text { ANCHO } \\
\text { (CM) }\end{array}$ & $\begin{array}{r}\text { AREA } \\
\text { SECCIÓN } \\
\text { (CM2) }\end{array}$ & $P(K N)$ & $\begin{array}{r}\sigma \text { ULT } \\
\text { (MPA) }\end{array}$ \\
\hline 1 & 1.41 & 1.15 & 1.62 & 23.23 & 143.26 \\
\hline 2 & 1.31 & 1.24 & 1.62 & 22.48 & 138.39 \\
\hline 3 & 1.45 & 1.15 & 1.67 & 23.50 & 140.93 \\
\hline 4 & 0.97 & 1.17 & 1. 3 & 15.48 & 136.40 \\
\hline 5 & 0.78 & 1.13 & 0.88 & 11.41 & 129.45 \\
\hline 6 & 1.1 & 1.13 & 1.24 & 17.24 & 138.70 \\
\hline 7 & 1.2 & 1.08 & 1.30 & 16.02 & 123.61 \\
\hline 8 & 0.99 & 1.18 & 1.17 & 18.84 & 161.27 \\
\hline 9 & 1.11 & 1.62 & 1.80 & 26.49 & 147.30 \\
\hline 10 & 1.24 & 1.09 & 1.35 & 17.37 & 128.51 \\
\hline 11 & 1.16 & 1.12 & 1.30 & 16.97 & 130.62 \\
\hline 12 & 1.18 & 1.14 & 1.35 & 19.93 & 148.16 \\
\hline
\end{tabular}

Fuente: Córdova, P. (2014). Obtención de las propiedades mecánicas y estructurales de la caña guadua Angustifolia Kunth del Ecuador.

TABLA 6. ANÁLISIS ESTADÍSTICO DEL ESFUERZO DE TRACCIÓN
ÓLTIMA DE CAÑA GUADUA
\begin{tabular}{lrl}
\hline o ULT MEDIA $=$ & 138.884 & Mpa \\
\hline Desviación estantadar $(S)=$ & 10.383 & Mpa \\
ok= & 117.598 & Mpa \\
S/ oult Media $=$ & 0.075 & \\
\hline
\end{tabular}

Fuente: Córdova, P. (2014). Obtención de las propiedades mecánicas y estructurales de la caña guadua Angustifolia Kunth del Ecuador. 


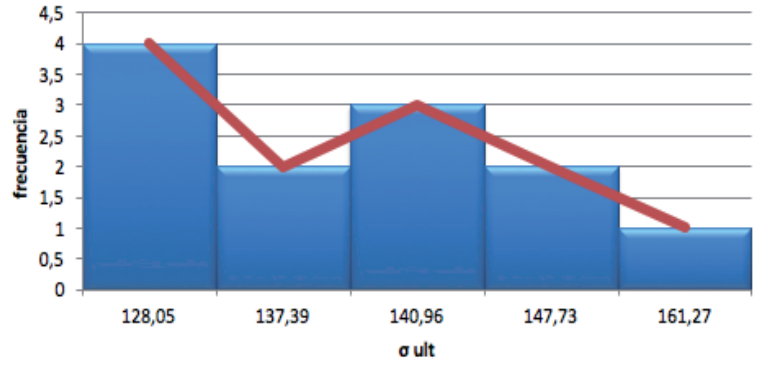

Figura 10. Análisis estadístico del esfuerzo a tracción paralela a la fibra última de la Janssen guadua.

Fuente: Córdova, P. (2014). Obtención de las propiedades mecánicas y estructurales de la caña guadua Angustifolia Kunth del Ecuador.

\begin{tabular}{|c|c|c|c|}
\hline $\begin{array}{l}\text { MUESTRA } \\
\text { N }\end{array}$ & $\begin{array}{l}0.5 \sigma \mathrm{ULT} \\
\text { (MPA) }\end{array}$ & $\mu \varepsilon(0.5 \mathrm{oULT})$ & $\begin{array}{l}=(0.5 \sigma \text { ULT }) /(E(0.5 \circ U L T)) \\
(G P)\end{array}$ \\
\hline 1 & 19.725 & 677 & 29.136 \\
\hline 2 & 25.747 & 1123 & 22.923 \\
\hline 3 & 28.616 & 962 & 29.736 \\
\hline 4 & 25.041 & 1379 & 18.162 \\
\hline 5 & 26.647 & 978 & 27.236 \\
\hline 6 & 26.262 & 988 & 26.575 \\
\hline 7 & 23.552 & 916 & 25.717 \\
\hline 9 & 21.506 & 852 & 25.248 \\
\hline 10 & 23.570 & 838 & 28.135 \\
\hline 11 & 22.594 & 1065 & 21.223 \\
\hline 12 & 24.748 & 683 & 36.231 \\
\hline 14 & 19.623 & 1229 & 15.973 \\
\hline
\end{tabular}

Fuente: Córdova, P. (2014). Obtención de las propiedades mecánicas y estructurales de la caña guadua Angustifolia Kunth del Ecuador.

TABLA 8. ANÁLISIS ESTADÍSTICO DEL MÓdULO DE ELASTICIDAD DE CAÑA GUADUA DE TRACCIÓN PARALELA A LA FIBRA

\begin{tabular}{lrl}
\hline E ULT MEDIA $=$ & 18.410 & $\mathrm{GPa}$ \\
\hline Desviación estantadar $(S)=$ & 4.811 & $\mathrm{GPa}$ \\
\hline Ek $=$ & 8.308 & $\mathrm{GPa}$ \\
$\mathrm{S} /$ Eult Media $=$ & 0.261 & \\
\hline
\end{tabular}

Fuente: Córdova, P. (2014). Obtención de las propiedades mecánicas y estructurales de la caña guadua Angustifolia Kunth del Ecuador.

Se obtuvo las curvas esfuerzo-deformación unitaria de diez muestras sometidas a tracción paralela a la fibra. En la figura 11 es apreciada la curva de la muestra 2. De estas curvas podemos concluir que la caña guadua sometida a tracción paralela a la fibra se comporta como un material elástico. Calculamos su módulo de elasticidad al 50\% de la carga última, resultando en $8308 \mathrm{MPa}$, el cual es muy bajo comparándolo con el MOE del acero, $206010 \mathrm{MPa}$.

El acero cuando está sometido a tracción con una carga igual a $117.6 \mathrm{MPa}$, sufre una deformación unitaria igual a 0.00057, mientras que cuando la caña guadua está sometida a su carga característica última a tracción, 117.6MPa, sufre una deformación unitaria igual a 0.011 , lo que quiere decir que la caña sufre mayor deformación para la misma carga. Por esta razón se debe prestar mucha atención a las deformaciones cuando se diseñen estructuras de caña guadua, puesto que van a sufrir muchas deformaciones cuando soporten cargas excesivamente pesadas. En la determinación del MOE se obtuvo una gran variedad de valores dispersos. Los resultados se muestran en las Tablas 7 y 8.

\section{(T2) $\sigma(\mathrm{Mpa}) / \delta$}

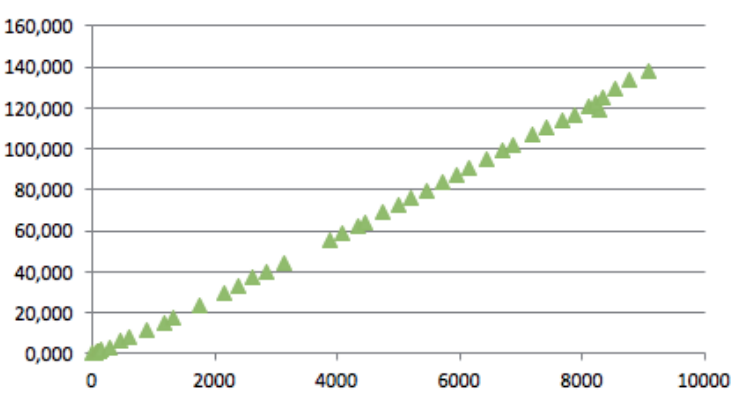

Figura 11. Curva del esfuerzo de tracción paralela a la fibra vs. la deformación unitaria de la muestra T2.

Fuente: Córdova, P. (2014). Obtención de las propiedades mecánicas y estructurales de la caña guadua Angustifolia Kunth del Ecuador.

\section{DISCUSIÓN DE RESULTADOS}

A partir del análisis estadístico realizado a los resultados, producto de los ensayos de resistencia al esfuerzo de compresión de caña guadua, se concluye que ésta es una propiedad mecánica muy resistente que tiene este tipo de caña, comparándola con el resto de los materiales que habitualmente se utilizan en construcción. Además se obtuvieron resultados bastantes uniformes en esta propiedad.

De los resultados de las pruebas de la resistencia a la compresión, se determina que a medida que el espesor de la pared de la caña, el diámetro exterior de la caña y el área sometida a compresión son mayores, mayor va a ser la resistencia al esfuerzo de compresión que va a tener la caña guadua. Así mismo, se determina que a medida que la relación Esp/Dext es mayor, 
la resistencia al esfuerzo de compresión va a ser mayor.

En el análisis estadístico hecho a los resultados de los ensayos de resistencia al esfuerzo de tracción, se concluye que, ésta es la propiedad mecánica más resistente que tienen la caña guadua, comparándola con el resto de sus propiedades mecánicas. Por lo que se puede concluir que la falla a tracción en un elemento estructural de caña guadua es la que menos probabilidad tiene de ocurrir.

Si se compara la resistencia a la tracción de la caña guadua con la del resto de materiales que habitualmente se utilizan en construcción, se considera que es muy alta. Es decir, la caña guadua es un material muy apto para la construcción utilizando elementos estructurales sometidos a tracción. Además se obtuvieron resultados uniformes en esta propiedad.

Analizando los resultados de los módulos de elasticidad de compresión y tracción se concluye que la Caña guadua puede ser considerada como un material con un comportamiento elásticofrágil de forma bilineal.

Para tener una resistencia mayor en elementos estructurales sometidos a tracción se debe tener un área mayor de las fibras sometidas a tracción; es decir, un mayor espesor de la pared de la caña guadua y un mayor diámetro externo de ésta.

Para futuras investigaciones se debe tomar en cuenta para un análisis estadístico de las propiedades geométricas de la caña guadua, la influencia de la conicidad, es decir, la forma de cono truncado que tiene por sus cambios de diámetro a lo largo de ésta.

La resistencia a compresión obtenida en este trabajo de investigación está limitada por el tamaño de las muestras, deberían hacerse estudios de compresión y de flexocompresión a muestras de caña guadua largas para determinar su comportamiento y resistencia como columnas.

\section{REFERENCIAS BIBLIOGRÁFICAS}

Amada, S., Untao, S. 2001. Fracture properties of bamboo. Part B 32 (2001) 451-459.

Asociación Colombiana de Ingeniería Sísmica, Normas Colombianas de diseño sismorresistente
Conference on Timber Engineering (2010) (NSR98), Asociación colombiana de Ingeniería Sísmica, Bogotá, Colombia, 1997.

Córdova, P. (2014). Obtención de las propiedades mecánicas y estructurales de la caña Guadua Angustifolia Kunth del Ecuador. (Trabajo de Grado). Facultad de Ingeniería, Universidad Católica de Santiago de Guayaquil, Guayaquil.

David J. A. Trujillo.(2009) Axially Loaded Connections In Guadua Bamboo. Proceedings of the Nocmat 2009, Bath, UK.

E. Dutta et. Al .(2008) Utilization aspects of bamboo and its market value. Indian Forest March-2008.

Fotografie Studio Zuarq - Bogotà www.zuarq. com.

Ghavami, K. et al. (2003). Bamboo: Functionally Graded Composite Material. Asian Journ. of Civil Eng.Vol. 4, No. 1.

Chavami, K. (2005). Bamboo as reinforcement in structural concrete elements. Cement \& Concrete Composites 27.

J. Coreal et at (2010). Structural Behavior Of Glued Laminated Guadua Bamboo as a Construction Material. World Conference on Timber Engineering 2010.

L. Osorio, E.Trujillo, A.W. Van Vuure, F. Lens, J. Ivens, I. Verpoest The Relation Between Bamboo Fibre Microstructure And Mechanical Properties. 14th European Conference On Composite Materials - Budapest, Hungary.

La Tegola, A., Mera, W. (2012). Diseño de las estructuras de hormigón armado a los estados límites. Universidad Católica de Santiago de Guayaquil.

T.M. Obermann, R. Laude. Bamboo Poles For Spatial And Light Structures. Bamboo Space Research Project Universidad Nacional de Colombia 166_OB.

T. Tada, K. Hashimoto \& A. Shimabukuro (2010). On characteristics of bamboo as structural materials in Challenges, Opportunities and Solutions in Structural Engineering and Construction Chafoori (ed.) .

Wan Tarmeze Wan Ariffin (2005). Numerical Analysis of Bamboo and Laminated Bamboo Strip Lumber. Research Project 2002-2005 - Sponsor Government of Malaysia.

Yao, W. \& Li, Z. (2003) Flexural behavior of bamboofiberreinforced mortarlaminates. Cement \& Concrete Research 33. 